\title{
A Importância do ensino de Libras: Relevância para Profissionais de Saúde
}

Tâmara Silva Ramos ${ }^{l}$; Maria Antonieta Pereira Tigre Almeida ${ }^{2}$

\begin{abstract}
Resumo: A audição é o sentido por meio do qual se percebem os sons e a deficiência auditiva por sua vez deve ser tema de discussão, pois embora seja direito da pessoa surda ter acesso a serviços de saúde de qualidade, são por muitas vezes, atendidos de maneira incorreta e desrespeitados em sua condição, pois a área de saúde não possui profissionais capacitados para um atendimento aprimorado. Assim este estudo teve por objetivo analisar a importância do estudo de Libras para uma melhor preparação profissional de saúde para atuar junto à comunidade surda, conforme questionários aplicados em um Universidade particular da cidade $\mathrm{cm}$ alunos de 5 tipos de áreas de saúde (Enfermagem, Farmácia, Odontologia, Fisioterapia e Estética) e por isso trata-se de uma pesquisa qualitativa onde o caráter é de maneira exploratória realizada com alunos dos cursos da saúde. Nota-se que Enfermagem, Fisioterapia, Farmácia, Estética e Odontologia. A maioria dos alunos cursam Enfermagem $(\mathrm{n}=22)$, 32 dos participantes sabem o que é Libras e não se comunicam por Libras. 36 participantes declararam ter esse interesse em estudar LIBRAS. E embora o cuidado e contato com algum paciente com problema auditivo, nesta pesquisa ter sido baixo $(n-5),(n=40)$ consideraram importante o estudo da libra na área de saúde e $(n=39)$ acreditam que todas as áreas de saúde deveriam ter disciplina de LIBRAS na grade curricular. Já que 34 deles disseram que o atendimento aos deficientes auditivos feito por sua classe é ruim. Chegando a conclusão de que as garantias e direitos dados às pessoas com deficiência devem existir como forma de inclusão e integração social.
\end{abstract}

Palavras Chaves: Surdo; Libras; Estudantes. Inclusão

\section{The Importance of Teaching Libras: Relevance for Healthcare Professionals}

\begin{abstract}
Is hearing the sense through which the perceived sounds and hearing impairment should in turn be a topic of discussion, for although it is the right of the deaf person to have access to quality health services, they are often taken care of incorrectly And disrespected in their condition, since the health area does not have professionals qualified for an improved care. Thus, this study aimed to analyze the importance of the study of Libras for a better professional health preparation to work with the deaf community, according to questionnaires applied in a private University of the city in students of 5 types of health areas (Nursing, Pharmacy, Dentistry, Physical Therapy and Aesthetics) and therefore it is a qualitative research where the character is in an exploratory way performed with students of the health courses. Note that Nursing, Physiotherapy, Pharmacy, Aesthetics and Dentistry. Most students attend Nursing $(\mathrm{n}=22), 32$ of the participants know what Libras are and do not communicate with Pounds. 36 participants stated that they had an interest in studying LIBRAS. And although the care and contact with a patient with a hearing problem in this research was low $(n-5),(n=40)$ considered important the study of the pound in the health area and $(n=39)$ believe that all areas Should have a discipline of LIBRAS in the curriculum. Since 34 of them said that the service for hearing impaired by their class is bad. It came to the conclusion that the guarantees and rights given to people with disabilities should exist as a form of inclusion and social integration.
\end{abstract}

Keywords: Deaf; Pounds; Students. Inclusion

\footnotetext{
${ }^{1}$ Graduanda do curso de Enfermagem da Faculdade Independente do Nordeste - FAINOR. E-mail: tati_.s2@hotmail.com;

${ }^{2}$ Professora Mestre do curso de Enfermagem da Faculdade Independente do Nordeste - FAINOR. Orientadora de Trabalhos de Conclusão de Curso.
} 
Id on Line Revista Multidisciplinar e de Psicologia

Id on Line Multidisciplinary and Psycology Journal

\section{Introdução}

A língua, a linguagem e a comunicação são fatores essenciais para que as interações sociais e relações interpessoais ocorram. E diante de tais premissas, os equipamentos sociais disponíveis são poucos para atender demandas de pessoas com deficiência, que precisam de um amparo especial (HAMZE, 2010).

Tal fato é agravado pelo fato de a sociedade não oferecer condições para que uma pessoa surda tenha a mesma oportunidade de acesso a linguagem, e neste contexto, a língua de sinais se torna meio propagador para efetivar a comunicação (DIZEU; CAPORALI, 2005).

$\mathrm{Na}$ área da saúde a comunicação do profissional com o paciente representa a principal maneira de criar vínculos com o paciente e familiares. Para que haja um eficaz comunicação entre profissionais da saúde e alguém surdo é necessário tomar medidas cabíveis para facilitar a linguagem, evitando barreiras (CHICON; SOARES, 2013).

Embora seja um direito da pessoa surda ter acesso a serviços de saúde de qualidade, estes são por muitas vezes, atendidos de maneira incorreta e até mesmo são, em alguns casos, desrespeitados em sua condição, pois há dificuldade na comunicação, já que os serviços de saúde não possuem profissionais capacitados para um atendimento de excelência a eles (SOUZA; PORROZZI, 2009).

Diante do contexto objetiva-se analisar a hipótese da Língua Brasileira de Sinais LIBRAS como disciplina obrigatória nos cursos de formação profissional na área da saúde, com a finalidade de maior interação entre profissional e paciente surdo, visando a melhoria do atendimento à saúde e ainda sendo relevante demonstrar o quão benéfico é melhorar a assistência da saúde primando pela diversidade e atendendo da melhor forma pessoas que precisam de atendimento especial (PAGLIUCA; FIÚZA,2007).

Nota-se que a maior parte dos profissionais de saúde não está preparado nem capacitado para se deparar com um portador de deficiência auditiva, isso muitas vezes gera um déficit na qualidade integral da assistência (AGUIAR; MARCUCCI, 2009).

Deste modo este estudo pretende destacar a necessidade da inclusão do ensino e libras como matéria obrigatória nos cursos deformação dos profissionais da saúde, e tem por objetivo analisar a importância do componente Libras na grade curricular para preparação profissional de saúde na atuação junto à comunidade surda. E especificamente visa realizar um estudo sobre o nível de conhecimento dos discentes, da área de saúde, no que se refere a comunicação com 
Id on Line Revista Multidisciplinar e de Psicologia

Id on Line Multidisciplinary and Psycology Journal

pessoa surda e ainda identificar as necessidades comunicativas dos profissionais de cada curso, da área de saúde, para desempenhar bem sua profissão.

\section{Metodologia}

Trata-se de uma pesquisa exploratória-descritiva que segundo Gil (2010) pretende proporcionar maior familiaridade com o tema de modo a explicitá-lo. Tida ainda como uma pesquisa qualitativa, com caráter descritivo, cujo objetivo primordial a descrição das características de determinadas população ou fenômenos ou, então, o estabelecimento de relação entre variáveis.

O local do presente estudo foi uma Faculdade Particular de Vitória da Conquista onde é oferecida a disciplina LIBRAS no curso de graduação. A amostra deste estudo abarcou 40 alunos dos cursos da saúde da Faculdade citada.

O material utilizado como instrumento de pesquisa e coleta de dados foi um questionário aplicado com os discentes, durante o mês de agosto de 2016, previamente aprovado pelo Comitê de Ética e Pesquisa da Faculdade Independente do Nordeste-FAINOR.

Salienta-se ainda que todos os participantes foram informados a respeito dos objetivos do estudo através de termo de consentimento esclarecido, obedecendo às recomendações da Resolução nº66/2012 do Conselho Nacional de Saúde (BRASIL, 2002b)

\section{Resultados e Discussões}

Na Tabela 1, constam-se os dados dos 40 entrevistados, todos, estudantes de uma Universidade particular da cidade de Vitória da Conquista- Bahia, englobando universitários de cinco cursos superiores: Enfermagem, Fisioterapia, Farmácia, Estética e Odontologia. Nota-se que mais da metade dos alunos são do curso de enfermagem $(n=22)$, sendo que seis deles são do curso de Farmácia e seis também do curso de Fisioterapia, 3 deles de Odontologia e 3 do curso de Estética. 
Com relação ao gênero, nota-se que 31 dos participantes são do sexo feminino e apenas 9 do sexo masculino.

A tabela 1 a seguir demonstra a distribuição relacionada a tais aspectos relacionados ao curso e gênero dos participantes da pesquisa

\begin{tabular}{|c|c|c|}
\hline \multicolumn{3}{|c|}{$\begin{array}{l}\text { TABELA 1. Distribuição dos dados referentes ao Curso e Gênero dos } \\
\text { participantes. Vitória da Conquista, Bahia, } 2016 .\end{array}$} \\
\hline \multirow[t]{2}{*}{ Curso e Gênero } & \multicolumn{2}{|c|}{ Total } \\
\hline \multirow{2}{*}{\multicolumn{3}{|c|}{ Curso }} \\
\hline & & \\
\hline Odontologia & 3 & 7,5 \\
\hline Estética & 3 & 7,5 \\
\hline Farmácia & 6 & 15 \\
\hline Fisioterapia & 6 & 15 \\
\hline Enfermagem & 22 & 55 \\
\hline Total & 40 & 100 \\
\hline \multicolumn{3}{|l|}{ Gênero } \\
\hline Masculino & 9 & 22,5 \\
\hline Feminino & 31 & 77,5 \\
\hline Total & 40 & 100 \\
\hline
\end{tabular}

Fonte: Dados da Pesquisa

Vê-se que a maioria dos estudantes eram do Curso de Enfermagem e a grande maioria mulheres, tal fato, pode ser relacionado a questão que no Curso de Enfermagem e na área de saúde em geral é mais comum a presença de mulheres em proporção maior. Nas próprias universidades as mulheres predominam as cadeiras.

Lopes e Leal (2005) destacam em seu estudo sobre a feminização persistente que ocorre na enfermagem se dá principalmente pelo fato da enfermagem trabalhar com o cuidado ao paciente, função essa mais atrelada ao gênero feminino.

A tabela 2 a seguir demonstra a distribuição relacionada aos aspectos relacionados ao tema Libras, conforme os questionários aplicados com alunos de Cursos da Área de saúde de uma Faculdade particular da cidade de Vitória da Conquista/Ba: 


\begin{tabular}{|c|c|c|}
\hline \multicolumn{3}{|l|}{$\begin{array}{l}\text { TABELA 2. Distribu } \\
\text { Bahia, Brasil, } 2016 .\end{array}$} \\
\hline \multirow[t]{2}{*}{ Sobre LIBRAS } & \multicolumn{2}{|c|}{ Total } \\
\hline & & $\%$ \\
\hline \multicolumn{3}{|l|}{ Sabe o que é Libras } \\
\hline Sim & 32 & 80 \\
\hline Não & 2 & 5 \\
\hline Um pouco & 6 & 15 \\
\hline Total & 40 & 100 \\
\hline \multicolumn{3}{|l|}{ Utiliza Libras } \\
\hline Sim & 4 & 10 \\
\hline Não & 32 & 80 \\
\hline Um pouco & 4 & 10 \\
\hline Total & 35 & 100 \\
\hline \multicolumn{3}{|c|}{ Interesse em estudar Libras } \\
\hline Sim & 36 & 90 \\
\hline Não & 4 & 10 \\
\hline Total & 40 & 100 \\
\hline \multicolumn{3}{|c|}{ Já tiveram contato com paciente surdo } \\
\hline Sim & 5 & 12,5 \\
\hline Não & 35 & 87,5 \\
\hline Total & 40 & 100 \\
\hline \multicolumn{3}{|c|}{ Importante o ensino de LIBRAS para profissionais de saúde } \\
\hline Sim & 40 & 100 \\
\hline Não & 0 & - \\
\hline Total & 40 & 100 \\
\hline \multicolumn{3}{|c|}{ Libras deve estar na grade curricular dos cursos de saúde } \\
\hline Sim & 39 & 97,5 \\
\hline Não & 1 & 2,5 \\
\hline Total & 40 & 100 \\
\hline \multicolumn{3}{|c|}{ Como sua classe atende o deficiente auditivo } \\
\hline Bom & 0 & 0 \\
\hline Moderado & 6 & 15 \\
\hline Ruim & 34 & 85 \\
\hline Total & 40 & 100 \\
\hline
\end{tabular}

Fonte: Dados da Pesquisa

Nota-se com relação à pesquisa sobre libras, que 32 dos participantes sabem o que é LIBRAS, 6 deles disseram não saber o que é, e 2 disseram saber um pouco sobre esse assunto.

Estudo feito por Aguiar e Marcucci (2009) destacam a necessidade das pessoas buscarem formas de comunicação e no caso as do tipo não verbal são essenciais e alerta para o fato de que as LIBRAS deveria ser estipulado de forma mais efetiva.

Quando questionados se conhecem ou utiliza a linguagem de libras, nota-se que 4 dos participantes disseram que sim, 4 disseram que um pouco, e a grande maioria $(n=32)$ disseram que não. 
Id on Line Revista Multidisciplinar e de Psicologia

Id on Line Multidisciplinary and Psycology Journal

Neste contexto, destaca-se que poucas pessoas usam as Libras e que a comunicação com pacientes surdos é facilitada pelo conhecimento desta língua de sinais, porque de forma que tanto o profissional quanto o paciente poderão ser compreendidos e assim será estabelecida uma relação de confiabilidade, desta maneira o paciente terá mais segurança para procurar os serviços de saúde.

Segundo Bortagarai e Ramos (2012) seja qual for a forma de comunicação, verbal ou não verbal, ela sempre deve estar presente na cena terapêutica, veiculando conteúdos conscientes e inconscientes, cuja significação está vinculada ao contexto em que ocorre.

Chaveiro e Barbosa (2005) quando tratam do tema acrescentam que compromisso social peca com as pessoas com deficiência. Alertando que a sociedade não está preparada para ao acolhimento, tendo como resultado uma educação que resultará na má preparação profissional, muito menos com a ética social.

Com relação ao interesse de estudar LIBRAS, enfatiza-se que 36 participantes declararam ter esse interesse, enquanto apenas 4 disseram que não tem interesse em estudar Libras.

O grupo de profissionais de assistência à saúde deve apresentar uma capacitação para o atendimento de pacientes surdos. O conhecer LIBRAS é de fundamental importância para todos os profissionais da saúde, uma vez que deveria ser componente curricular obrigatório na graduação profissional. Nota-se neste estudo que a maioria tem interesse em aprender essa linguagem.

Tal fato se dá porque o deficiente auditivo necessita de cuidado especial, para a inclusão social, no sentido de dar a eles o necessário para que fiquem em condições igualitárias com qualquer pessoa e não sejam prejudicados em nenhum momento, e isso se dá através do uso de recursos necessários para acabar com as barreiras no processo de atendimento e acesso à saúde respeitando todos seus direitos, através da cidadania (HAMZE, 2010).

Ainda com relação a está temática, estudo de Aguiar e Marcucci (2009) trata que acadêmicos e profissionais na área da saúde, devem buscar adquirir conhecimento para a manter uma comunicação não-verbal, facilitando a interlocução com o deficiente auditivo

Desse modo, vale acrescer ainda que quando questionados se já teve contato com algum paciente com problema auditivo, nota-se que 5 participantes disseram que sim, enfatizando que dois desses do curso de Farmácia, dois de Fisioterapia e um de Enfermagem. 
Id on Line Revista Multidisciplinar e de Psicologia

Id on Line Multidisciplinary and Psycology Journal

Logo, nota-se que o lidar com pacientes deficientes auditivos é comum, pois os alunos sequer já estão na atuação profissional e já lidaram com pacientes que usavam a linguagem de sinais.

$\mathrm{Na}$ área da saúde a comunicação do profissional com o paciente representa a principal maneira de criar vínculos com o paciente e familiares. Para que haja um eficaz comunicação entre profissionais da saúde e surdo é necessário tomar medidas cabíveis para facilitar a linguagem, pois não deve haver barreiras físicas, econômicas e sociais impostas pelo ambiente ao indivíduo que tem deficiência (CHICON; SOARES, 2013).

Sobre se acha importante o ensino de LIBRAS para profissionais de saúde, nota-se que a todos participantes alegaram que $\operatorname{sim}(n=40) . E(n=39)$ destes acreditam que todas as áreas de saúde deveriam ter disciplina de LIBRAS na grade curricular.

Percebe-se assim, como traz Dessen e Brito (2007) que as pessoas com deficiência, neste caso com perda auditiva necessitam de um amparo especial, e a inclusão social é a melhor forma de fazer com que estas se sintam aptas socialmente para agir como uma pessoa que não tivesse limitações.

É necessário que os profissionais da Saúde, principalmente aqueles participantes da Atenção Básica e da Saúde da Família apresentem capacitação para se comunicarem de forma eficaz com tais pacientes (TEDESCO; JUNGES, 2013).

De acordo com Souza e Porrozzi (2009) tal capacitação se concretizaria quando houvesse o cursos de LIBRAS (Língua Brasileira de Sinais) implementado como política inclusiva e de integração e principalmente aplicado na área da educação e da saúde, para que os profissionais de tais áreas, pelo menos tenham um mínimo de entendimento necessário para o atendimento destas pessoas.

Tem sido enorme desafio para as instituições de ensino superior formar profissionais que não representem apenas fonte de transmissão de conhecimentos, mas, sobretudo, de atitudes e práticas inovadoras voltadas a valorização diversidade humana (GLAT; PLETSCH, 2010).

Com relação a como o questionado acha que sua classe profissional atende o paciente surdo, nota-se que 34 deles disseram que o atendimento é ruim e 6 deles julgam ser moderados, nenhum alega ser bom.

Nota-se que há necessidade de que as classes profissionais da área de saúde atentem para melhorar o atendimento a deficientes, ajudando-os na relação entre o portador de surdez 
Id on Line Revista Multidisciplinar e de Psicologia

Id on Line Multidisciplinary and Psycology Journal

com profissionais de saúde, efetivando melhor a linguagem e comunicação (NOBREGA, et al, 2012).

Porém ainda existem algumas resistências com relação a este público, já que percebese que pacientes surdos utilizam o sistema de saúde de modo diferente dos pacientes ouvintes e por terem dificuldade representadas por medo, desconfiança e frustração acabam por muitas vezes deixando de ir, evitando a assistência médica (CHAVEIRO; PORTO, 2009).

Tedesco e Junges (2013) e Costa e Silva (2012) enfatizaram em seus estudos que o Estado atualmente obriga a todas as universidades que ofertam as licenciaturas a dispor da disciplina de Libras, linguagem brasileira de sinais, utilizada para a comunicação dos surdos, em se tratando dos docentes que já estão distribuídos na rede de ensino.

Salienta-se que a estes sobram poucas alternativas visto que o próprio Estado que obriga não dispõe de mecanismos que os capacite, cabendo aos professores buscarem métodos de capacitação, como as pós-graduações de Libras (BRASIL, 2005).

Ortigara e Trecocci (2009) acreditam que por ser extremamente difícil a comunicação entre o profissional de saúde e o surdo, é essencial o aprendizado da Língua Brasileira de Sinais. Por este motivo a disciplina oferecida deveria ter uma carga horaria maior para que os estudantes pudessem se preparar melhor e se sentirem mais seguros para atender o paciente surdo.

Nota-se que as pessoas com deficiência, neste caso em discussões com perda auditiva necessitam de um amparo especial, e a inclusão social é a melhor forma de fazer com que estas se sintam aptas socialmente para agir como uma pessoa que não tivesse limitações, e a sociedade deve buscar para que medidas neste sentido sejam impostas em prol de pessoas que tem todos os direitos e capacidades de estarem em sociedade sem sofrer qualquer limitação frente a estes direitos (HAMZE, 2010).

\section{Considerações Finais}

Esse tema apresenta relevância social, pois contribui para o aumento do debate científico e possibilita a realização de publicações científicas, já que há uma escassez de discussões e 
Id on Line Revista Multidisciplinar e de Psicologia

Id on Line Multidisciplinary and Psycology Journal

estudos acerca desta temática, influenciada pelo fato de ser um assunto de preconceito da sociedade que nem sempre ver as necessidades das pessoas com deficiência.

Observa-se que a inclusão de pessoas com deficiência, através da comunicação é uma necessidade alarmante, e é necessário que a sociedade forneça subsídios para atuar na área de saúde com a possibilidade de transformação do sujeito de maneira que este seja respeitado, focando sobre a proteção social, efetivando a dignidade que pode ser alcançada.

Por ser uma pesquisa firmada em análise de questionário viu-se que a maioria dos pesquisados se interessam pelo tema e acham importante a sua incorporação nas grades e metodologias de ensino na área de saúde, para que melhore a assistência a essas pessoas.

Deve-se implementar um conhecimento científico nas equipes de saúde, na compreensão das ações desenvolvidas em relação as principais dificuldade que o profissionais de saúde enfrentam diante a assistência ao paciente com dificuldade auditiva. E a sociedade deve buscar para que medidas neste sentido sejam impostas em prol de pessoas que tem todos os direitos e capacidades de estarem em sociedade sem sofrer qualquer limitação frente a estes direitos.

\section{Referências}

AGUIAR, Fernanda S.; MARCUCCI, Rosa Maria B. Uso da linguagem brasileira de sinais na comunicação enfermeiro paciente portador de deficiência auditiva. Rev. Enferm UNISA, 10(2): 144-8, 2009. Disponível em: <http://www.unisa.br/graduacao/biologicas/enfer/revista/arquivos/2009-2-08.pdf. > Acesso em 04 out 2016.

BORTAGARAI F., RAMOS A. A comunicação não-verbal na área da saúde, Rev. CEFAC, São Paulo, 2012. Disponível em: < http://www.scielo.br/pdf/rcefac/2011nahead/186_10.pdf> Acesso em 04 out 2016.

BRASIL. Decreto $n^{0}$ 5.626, de 22 de dezembro de 2005. Regulamenta a Lei no 10.436, de 24 de abril de 2002, que dispõe sobre a Língua Brasileira de Sinais - Libras, e o art. 18 da Lei no 10.098, de 19 de dezembro de 2000. Brasília: Ministério da Justiça, 2005. Disponível em:< http://r1.ufrrj.br/graduacao/arquivos/docs_academico/decreto_5626_libras.pdf. >Acesso em 20 mai 2016. 
Id on Line Revista Multidisciplinar e de Psicologia

Id on Line Multidisciplinary and Psycology Journal

CHAVEIRO, Neuma; BARBOSA, Maria Alves. Assistência ao surdo na área de saúde como fator de inclusão social. Rev. esc. enferm. USP, São Paulo, v. 39, n. 4, p. 417-422, Dec. 2005. Disponível em: <<http://www.scielo.br/scielo.php?script=sci_arttext\&pid=S0080$62342005000400007 \& \operatorname{lng}=e n \& n r m=i s o>$. Acesso em 23 set 2016.

CHAVEIRO, Neuma; PORTO, Celmo Celeno; BARBOSA, Maria Alves. Relação do paciente surdo com o médico. Rev. Bras. Otorrinolaringol., São Paulo , v. 75, n. 1, p. 147150, Feb. 2009. Disponível em:<

http://www.scielo.br/scielo.php?script=sci_arttext\&pid=S0034-

$72992009000100023 \& \operatorname{lng}=$ en\&nrm=iso $>$ Acesso em 20 out 2016.

CHICON. José Francisco; SOARES. Jane Alves. Compreendendo os Conceitos de Integração e Inclusão. 2013. Disponível em: < http://www.todosnos.unicamp.br:8080/ lab/links-uteis/acessibilidade-e-inclusao/textos/compreendendo-os-conceitos-de-integracao-einclusao/> Acesso em 04 nov 2016.

COSTA, Luiza Santos Moreira da; SILVA, Natália Chilinque Zambão da. Desenvolvendo atitudes, conhecimentos e habilidades dos estudantes de medicina na atenção em saúde de pessoas surdas. Interface (Botucatu), Botucatu , v. 16, n. 43, p. 1107-1117, Dec. 2012.

DESSEN. Maria Auxiliadora; BRITO. Angela Maria Waked de. Reflexões sobre a deficiência auditiva e o atendimento institucional de crianças no Brasil. 2007. Disponível em: < http://www.scielo.br/pdf/paideia/n12-13/09.pdf > Acesso em 05 jun 2016.

DIZEU, Liliane Correia Toscano de Brito; CAPORALI, Sueli Aparecida. A língua de sinais constituindo o surdo como sujeito. Educ. Soc., Campinas , v. 26, n. 91, p. 583-597, Aug. 2005. Disponível em: <http://www.scielo.br/scielo.php?script=sci_arttext\&pid=S0101$73302005000200014 \& \operatorname{lng}=\mathrm{en} \& n \mathrm{~nm}=\mathrm{iso}>$. Acesso em 07 jun 2016.

GIL, A. C. Como Elaborar Projetos de Pesquisa. 6ª Ed. São Paulo. Editora: Atlas, 2010.

GLAT, R.; PLETSCH, M. D. O papel da Universidade no contexto da política de Educação Inclusiva: reflexões sobre a formação de recursos humanos e a produção de conhecimento. Rev. Educ. Espec, Santa Maria, v. 23, n. 38, p. 345-356, 2010. Disponível em: <http://www.ufsm.br/revistaeducacaoespe> Acesso em 03 jun 2016.

HAMZE. Amelia. Integração ou Inclusão? 2010. Disponível em: < http://educador.brasilescola.com/trabalho-docente/integracao.htm> Acesso em 03 jun 2016. 
Id on Line Revista Multidisciplinar e de Psicologia

Id on Line Multidisciplinary and Psycology Journal

LOPES. Marta Júlia Marques; LEAL. Sandra Maria Cezar. A feminização persistente na qualificação profissional da enfermagem brasileira. Cadernos pagu (24), janeiro-junho, 2005, pp.105-125.

NOBREGA, Juliana Donato et al . Identidade surda e intervenções em saúde na perspectiva de uma comunidade usuária de língua de sinais. Ciênc. saúde coletiva, Rio de Janeiro, v. 17, n. 3, p. 671-679, Mar. 2012.

ORTIGARA, Elisangela P. F.; TRECOSSI, Micheli O. Importância e eficácia das consultas de enfermagem ao paciente surdo. Revista de Enfermagem. Vol.9, n.9, p.60-69, 2013. Disponível em: <http://revistas.fw.uri.br/index.php/revistadeenfermagem /article/view/938. > Acesso em 01 jun 2016.

PAGLIUCA, L. M. F.; FIÚZA, N. L. G.; REBOUÇAS, C. B. A. Aspectos da comunicação da enfermeira com o deficiente auditivo. RevEscEnferm USP,Fortaleza, v.41, n.3, p.411-8, 2007. Disponível em:http://www.scielo.br/pdf/reeusp/v41n3/10.pdf Acesso em 20 mai 2016.

SOUZA, M. T.;PORROZZI, R. Ensino de libras para profissionais de saúde: uma necessidade premente. Rev. Práxis, v.1, n. 2, p.43-6, 2009.

TEDESCO, Janaina dos Reis; JUNGES, José Roque. Desafios da prática do acolhimento de surdos na atenção primária. Cad. Saúde Pública, Rio de Janeiro , v. 29, n. 8, p. 1685-1689, Aug. 2013 .

Como citar este artigo (Formato ABNT):

RAMOS, T.S.; ALMEIDA, M.A.P.T. A Importância do ensino de Libras: Relevância para Profissionais de Saúde. Id on Line Revista Multidisciplinar e de Psicologia, Janeiro de 2017, vol.10, n.33, p. 116-126. ISSN: 19811179 .

Recebido: 25/11/2016

Aceito: $25 / 11 / 2016$ 